\title{
Editorial
}

\section{Normes et société}

Hasard des dates, c'est au moment où je m’apprête à rédiger cet éditorial qu'est publiée dans le journal Le Soir une interview provocatrice du sociologue Claude Javeau ${ }^{1}$. Héraut des sociologies post-marxistes il nous rappelle, à l'occasion de la sortie de son dernier livre intitulé simplement Les paradoxes de la postmodernité, que l'individu n'est pas autonome. Ressuscitant l'Aliénation marxiste, il nous dresse un portrait fort pessimiste d'un processus constitutif de toutes sociétés : l'adéquation des pratiques individuelles à des normes collectives.

C'est justement cette question que nous proposons d'utiliser comme fil rouge tout au long de ce numéro. L'étude des normes sociales, des actions individuelles et de leurs rapports a toujours été présente dans les sciences sociales. Des fondateurs jusqu'aux sociologies constructivistes, la notion de norme a été employée pour comprendre et décrire les activités humaines. Les trois articles que nous présentons ne font pas exception, mais donnent tous trois un point de vue original sur cette question.

L'étude de Sophie Massot (doctorante à Science Po Paris) sur les Toy, fêtes extraordinaires qui prennent place en Ouzbékistan, nous montre comment l'ethnologue peut, en observant une situation extra-ordinaire, mettre au jour les normes constitutives de la vie quotidienne d'un groupe social.

En s'interrogeant sur le statut "d'artiste officiel" et la carrière du sculpteur Raymond Delammare, Claire Maingon (docteur en histoire de l'art) nous invite à nous interroger sur le rapport entre liberté de pratique et conventionalité artistique. Question paradoxale dans le domaine de l'art où les standards actuels de validité posent l'originalité comme base. Il n'est pas inutile de se rendre compte que les vérités de notre époque n’ont pas toujours été comme elles le sont.

La question de la normalité affleure enfin de manière très touchante dans les pages de Mathieu Simonson. Il aborde en effet la question polémique de l'avortement lors de la détection néo-natale d'une trisomie 21. Il renouvelle le débat en l'articulant à une approche

\footnotetext{
${ }^{1}$ Le Soir du 4 octobre 2007, page 21 : «L'individu postmoderne est un mouton de Panurge ». 
spinoziste originale et, tout en se gardant de donner une impossible solution clef sur porte, il répète l'importance d'une information disponible et clairement identifiable par rapport à la norme sociale.

Trois articles suffisent pour s'en rendre compte : la notion de norme renvoie à des idées et des réalités qui peuvent être très différentes. Rien ne sert cependant de réveiller les démons marxistes: la richesse des philosophies contemporaines françaises en anglo-saxonnes devrait permettre de préciser mieux que jamais la notion de norme sociale et par là même, d'amener à une meilleure compréhension de nos sociétés. C'est un défi qui mérite certainement d'être saisi...

Grégoire Lits, pour le comité de rédaction 